\title{
Ibuprofen induced Stevens-Johnson syndrome and liver injury in children: a case report
}

\author{
Ming-Zhu Gui ${ }^{1,2 \#}$, Ming Ni ${ }^{2,3 \#}$, Xue-Dong Yin ${ }^{2}$, Ting Zhang ${ }^{4}$, Zhi-Ling Li $^{2}$ \\ ${ }^{1}$ Department of Pharmacy, Shanghai Children's Hospital, Shanghai Jiao Tong University, Shanghai, China; ${ }^{2}$ Department of Pediatrics, Luodian \\ Hospital, Shanghai, China; ${ }^{3}$ Department of Clinical Pharmacy, Shaoxing Maternity and Child Health Care Hospital, Shaoxing, China; ${ }^{4}$ Department \\ of Gastroenterology, Shanghai Children's Hospital, Shanghai Jiao Tong University, Shanghai, China \\ \#These authors contributed equally to this work. \\ Correspondence to: Zhi-ling Li; Ting Zhang. Shanghai Children's Hospital, Shanghai Jiao Tong University, 355, Luding Road, Shanghai 200040, \\ China. Email: lizhiling22@163.com; zhangting@shchildren.com.cn.
}

\begin{abstract}
Stevens-Johnson syndrome (SJS) is a disorder that causes severe damage to the skin and mucous membranes with bullous and erosive properties. Drug-induced liver injury (DILI) is closely related to non-steroidal anti-inflammatory drugs (such as ibuprofen). Liver injury caused by ibuprofen is often related to overdose, and liver injury caused by normal dose is rare, and there are individual differences in different situations. In this case, a child developed SJS and acute liver injury after treatment with ibuprofen suspension. We described the characteristics of related adverse reactions induced by ibuprofen, and analyzed the relationship between SJS caused by the drug and related drug genes. Glucocorticoids and antihistamines were used to treat dermatitis, reduced glutathione $(\mathrm{GSH})$ to protect the liver and plasma exchange detoxification. Finally, the patient's dermatitis healed and the liver injury was significantly improved. Many studies have suggested that DILI may be related to human leukocyte antigen (HLA) genotyping. The detection of drug-related genes revealed that the SJS and liver damage caused by ibuprofen might have been related to the positive HLA-B*5801. This article suggests that attention should be paid to checking liver function indicators after taking ibuprofen, and genetic screening can be used to reduce the risk of generelated adverse reactions when necessary.
\end{abstract}

Keywords: Case report; Stevens-Johnson syndrome (SJS); liver damage; drug gene testing

Submitted Jan 07, 2021. Accepted for publication Apr 23, 2021.

doi: $10.21037 /$ tp-21-8

View this article at: http://dx.doi.org/10.21037/tp-21-8

\section{Introduction}

Ibuprofen, organically known as (2RS)-1-(4-[2-methyl propyl])phenyl, is a common over-the-counter analgesic with good anti-inflammatory and antipyretic properties. It is a propionic acid derivative and is classified as a nonsteroidal anti-inflammatory drug (NSAID) (1). The NSAIDs exert anti-inflammatory, analgesic, and antipyretic effects by suppressing prostaglandin (PG) synthesis by inhibiting the enzyme cyclooxygenase (COX).

Ibuprofen is widely used in children because of its rapid fever reduction and good curative effects. The incidence of DILI is often closely related to ibuprofen, but the prevalence of DILI caused by ibuprofen is not high (2). It is generally believed that it is one of the safest antipyretic drugs, and the drug has the highest benefit/ risk ratio. Nevertheless, when evaluating potential cases of hepatotoxicity, ibuprofen-related DILI also requires full attention, which usually manifests as liver cell damage. Some adverse drug reactions (ADR) have been reported recently in the literature, such as gastrointestinal tract irritation, peptic ulcer, high level of transaminase, and rash. Bronchospasm and allergic nephritis are among other relatively rare adverse reactions. Liver cell injury is the most common type of disease. Severe patients can even develop into acute liver failure leading to death. 


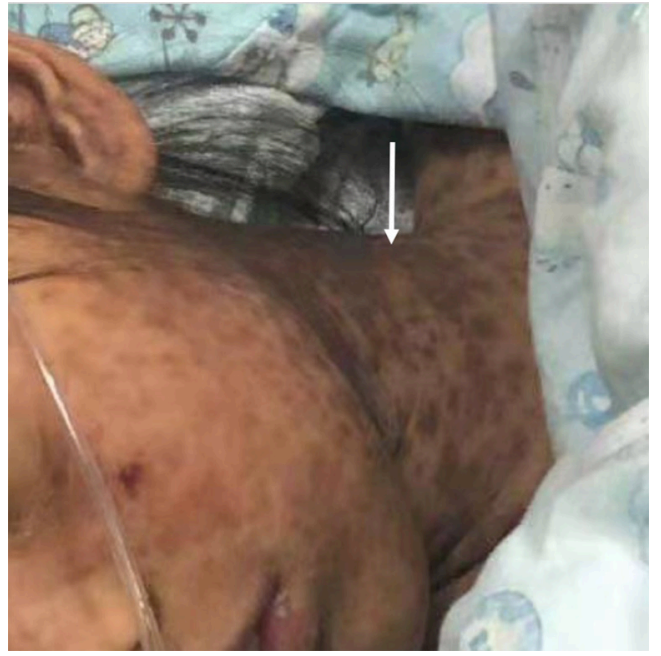

Figure 1 A small red rash appeared on the neck and then spread to the face and body. As shown by the white arrow, there is a large area of bullous erythema multiforme.

Stevens-Johnson syndrome (SJS) is a serious adverse skin reaction, but it is relatively rare, with an annual incidence of only $1-2 / 1,000,000$, which is regarded as a medical emergency. NSAIDs are listed as high-risk drugs to induce SJS (3).

This article described a child who developed SJS and liver injury after taking ibuprofen suspension (Johnson \& Johnson, H19991011) and discussed the case combined with the literature. We also summarized the experience of diagnosing and treating severe adverse reactions to Ibuprofen to improve medical staff understanding of ADR and strengthen the clinical application of pharmacogenomics.

We present the following case following the CARE reporting checklist (available at http://dx.doi.org/10.21037/ tp-21-8).

\section{Case presentation}

Female, 9 years old, the child has no previous special medical history, no concomitant diseases, no history of drug allergy, no liver-related diseases, and no exposure to suspicious drugs before and after the onset of the disease. This child (weight, $27 \mathrm{~kg}$ ) developed fever without obvious inducement, with the highest temperature of $38.6^{\circ} \mathrm{C}$, on 4 July 2019. She had taken ibuprofen suspension 3 times and had no abdominal pain, rash, vomiting, diarrhea, or cough at the beginning of the disease. Unfortunately, we could not determine the time and dose of ibuprofen at that time because the child was not seen in our hospital at that time. Since July 6, the patient developed somnolence, anorexia, abdominal pain, and a small red rash on the neck (Figure 1). At this time, stop ibuprofen and related drugs immediately. She attended Wuxi Children's Hospital for treatment, and her liver function was shown as abnormal by alanine aminotransferase (ALT, $1416 \mathrm{U} / \mathrm{L}$ ), aspartate aminotransferase (AST, $934 \mathrm{U} / \mathrm{L}$ ), total bilirubin $(68.4 \mu \mathrm{mol} / \mathrm{L})$, total bile acids $(345.1 \mu \mathrm{mol} / \mathrm{L})$ and $\gamma$-glutamyltransferase (669 U/L). Reducing GSH and acetylcysteine was given to protect the liver and lower enzymes. Recurrent abdominal pain persisted, rashes progressively worsened (involving the face, neck, and trunk), and the skin had begun to peel off. From 8 July, she was given cefpiramide sodium, ornidazole, imipenem, and cilastatin for anti-infection and gamma bulb support treatment. She was transferred to our hospital on 11 July 2019 due to "fever for 1 week, abdominal pain, rash, and abnormal liver function for 5 days". Upon admission examination, the patient presented the following: body temperature: $37.8^{\circ} \mathrm{C}$, pulse rate 93 beats $/ \mathrm{min}$, breathing 24 beats/min, blood pressure: 100/55 $\mathrm{mmHg}$. She was lucid, in good spirits, had no cyanosis of the lips, was showing dense brown skin rashes all over the body (face, neck, and trunk), which did not fade when pressed, an area of peeling on the chest about $10 \mathrm{~cm} \times 10 \mathrm{~cm}$ with no obvious exudation or suppuration, the neck was soft and without resistance, coarse breath sounds in both lungs without rales, uniform heart rhythm, strong heart sounds, reachable level II/III murmurs, flat and soft abdomen, liver palpable $3 \mathrm{~cm}$ below ribs, soft texture, spleen not palpable below ribs, physiological reflexes were present, the pathological reflex was not elicited. The laboratory analyses were as follows: white blood cell (WBC), $122.00 \mathrm{~g} / \mathrm{L}$; hemoglobin, $212.00 \times 10^{9} / \mathrm{L}$; neutrophils, $59.5 \%$; lymphocytes, $29.4 \%$; C-reactive protein, $6.2 \mathrm{mg} / \mathrm{L}$. Fecal routine + occult blood testing revealed the following: WBC microscopy $(+)$, occult blood (+). On July 13, 2019, Abdominal magnetic resonance imaging (MRI) revealed that: the liver was slightly enlarged, and the gallbladder was enlarged. A diagnosis was made of liver insufficiency, SJS, and an intestinal infection.

On the 3rd day of admission (July 13), the patient's liver function was aggravated according to dynamic monitoring, the direct bilirubin had increased, and jaundice had worsened (July 13, direct bilirubin, $169.69 \mu \mathrm{mol} / \mathrm{L}$; total bilirubin $187.07 \mu \mathrm{mol} / \mathrm{L}$; ALT, $283 \mathrm{U} / \mathrm{L}$; AST, $159 \mathrm{U} / \mathrm{L}$; $\gamma$-glutamyltransferase, $437 \mathrm{U} / \mathrm{L}$; total bile acid, $262 \mu \mathrm{mol} / \mathrm{L}$ ). The liver function of the patient during admission is shown in Table 1. 
Table 1 Changes of liver function

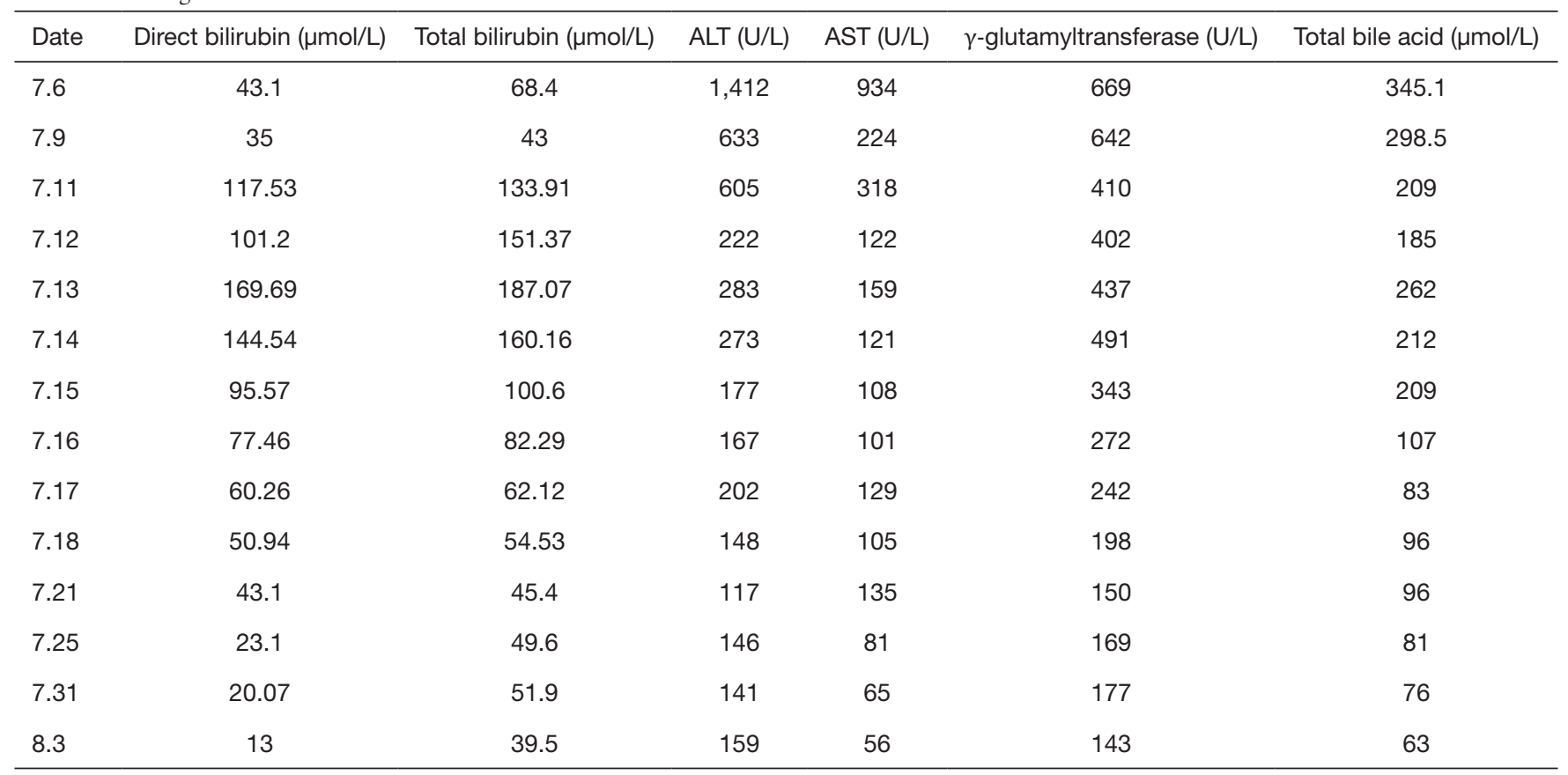

ALT, alanine aminotransferase; AST, aspartate aminotransferase.

Table 2 Detection of HLA genes related to adverse drug reactions

\begin{tabular}{ll}
\hline Test gene & Results \\
\hline HLA-B 5801 & Positive \\
\hline
\end{tabular}

HLA, human leukocyte antigen.

Before and after detecting the hepatitis $\mathrm{B}$ virus and hepatitis $\mathrm{C}$ antigen antibodies in children, the cytomegalovirus and rubella virus antibody tests did not indicate viral infection, and viral hepatitis was ruled out. Eosinophils were checked on admission and there was no high level of eosinophils. On July 30 , the drug-gene test result was positive for human leukocyte antigen (HLA)-B*5801, suggesting that the HLA-B ${ }^{*} 5801$ allele was carried. The genetic test results are shown in Table 2. Considering the severe exudative erythema multiforme (SJS) and cholestatic liver caused by drug damage (2). On August 3, direct bilirubin $13 \mu \mathrm{mol} / \mathrm{L}$, total bilirubin $39.5 \mu \mathrm{mol} / \mathrm{L}$, ALT $159 \mathrm{U} / \mathrm{L}$, AST $56 \mathrm{U} / \mathrm{L}, \gamma$-glutamyltransferase $143 \mathrm{U} / \mathrm{L}$, total bile acid $63 \mu \mathrm{mol} / \mathrm{L}$, Under the treatment plan of this hospital, the patient was discharged after the condition had gradually stabilized. No major complaints remained on the 15 th and 30th days of follow-up.
After admission, methylprednisolone was administered for 1 week, and loratadine for 3 weeks for anti-inflammatory, anti-allergic, and immunomodulating therapy. Reduced GSH and adenosylmethionine succinate were administered for 2 weeks, compound glycyrrhizin for 3 weeks, and ursodeoxycholic acid for 4 weeks to protect the liver and promote bile excretion, then plasma exchange was given twice (July 13 to $14,1,400 \mathrm{~mL} /$ time). The use time and dosage of the drug are shown in Figure 2. Mupirocin ointment (SK\&F) was applied to the wound, accompanied by gamma globulin, and albumin supportive treatments. The patient's condition and medication are shown in Figure 3.

All procedures performed in studies involving human participants were in accordance with the ethical standards of the institutional and/or national research committee(s) and with the Helsinki Declaration (as revised in 2013). Written informed consent was obtained from the patient.

\section{Discussion}

SJS is a rare and severe disease, a serious erythema multiforme, characterized by bullous and erosive damage to the skin and mucous membranes. It usually occurs after consuming nonsteroidal drugs, sulfa drugs, penicillin, allopurinol, and anticonvulsants (3). According to the 
$\begin{array}{llllllllllllllllllllllllll}11 & 12 & 13 & 14 & 15 & 16 & 17 & 18 & 19 & 20 & 21 & 22 & 23 & 24 & 25 & 26 & 27 & 28 & 29 & 30 & 31 & 1 & 2 & 3 & 4 & 5\end{array}$

\begin{tabular}{|c|c|c|c|c|c|c|c|c|c|c|c|c|c|c|c|c|c|c|c|c|c|c|c|c|c|c|c|}
\hline Purpose of medication & Drugs $\quad$ Admission day & 1 & 2 & 3 & 4 & 5 & 6 & 7 & 8 & 9 & 10 & 11 & 12 & 13 & 14 & 15 & 16 & 17 & 18 & 19 & 20 & 21 & 22 & 23 & 24 & 25 & 26 \\
\hline Anti-inflammatory & Methylprednisolone & \multicolumn{6}{|c|}{$30 \mathrm{mg}$ ivgtt. QD } & & & & & & & & & & & & & & & & & & & & \\
\hline Anti-allergic & Loratadine & \multicolumn{22}{|c|}{$10 \mathrm{mg}$ Po. QN } & & & & \\
\hline \multirow{3}{*}{ Protect the liver } & Reduced GSH & \multicolumn{12}{|c|}{$0.6 \mathrm{~g}$ ivgtt. QD } & & & & & & & & & & & & & & \\
\hline & Adenosylmethionine succinate & \multicolumn{12}{|c|}{$0.5 \mathrm{~g}$ ivgtt.QD } & & & & & & & & & & & & & & \\
\hline & Compound glycyrrhizin & \multicolumn{15}{|c|}{$20 \mathrm{~mL}$ ivgtt. QD } & \multicolumn{8}{|c|}{1 tab. po. Tid } & & & \\
\hline Promote bile excretion & Ursodeoxycholic & \multicolumn{26}{|c|}{$0.25 \mathrm{~g}$ Po. Bid } \\
\hline
\end{tabular}

Figure 2 Medication for children in hospital.

time correlation between children's medication and the appearance of skin lesions, the possibility of combining SJS with other medications besides ibuprofen can be ruled out.

The disease SJS is a recognized immune complexmediated allergic reaction that affects all age groups. It has typical systemic, mucous membrane, and skin damage manifestations. Caproni et al. showed that (4) tumor necrosis factor- $\alpha(\mathrm{TNF}-\alpha)$ is strongly expressed in SJS lesions and plays an important role in the SJS immune response.

Some studies have reported a close genetic relationship between HLA alleles and drug allergy susceptibility $(5,6)$. These genetic associations may be drug-specific. For example, HLA-B*1502 is related to SJS induced by carbamazepine (6), HLA-B*55:01 is related to penicillin allergy (7), and HLA-B ${ }^{*} 5801$ is related to severe skin adverse reactions induced by allopurinol (6).

There is a clear correlation between HLA-B ${ }^{*} 5801$ and the severe skin reaction caused by related drugs, while in North Korea, the correlation among Japanese and European populations is relatively weak. The average carrying rate of HLA-B ${ }^{\star} 5801$ among Chinese is $8.8-10.9 \%$ (8). The average mutation carrier rates of the Caucasian, Asian, and African populations are $1-6 \%, 3-15 \%$, and $2-4 \%$, respectively (9). In this case, the patient carried the HLA-B ${ }^{*} 5801$ genes, it might be a risk factor, more studies should be performed. Therefore, those who carry HLA-B ${ }^{\star} 5801$ genes need to be aware of drug-induced SJS when taking nonsteroidal drugs, sulfa drugs, penicillin, allopurinol, and anticonvulsants. The drug treatment of such patients needs to be cautious; attempts must be made to detect drug genes, followed by analysis and administration of individualized drugs.

Liver damage caused by drugs is relatively common, because most drugs are metabolized in the liver, such as ibuprofen, acetaminophen and other non-steroidal antiinflammatory drugs. Diagnosis of drug-induced liver injury (DILI) is an uncertain process, requiring a high degree of understanding of the disease and careful exclusion of other causes of liver disease. DILI can usually be divided into two types according to the liver toxicity mechanism. One is dose-related and the other is dose-independent. However, in this case, it is the liver injury induced at normal doses, which only occurs in a small part of the population. Idiosyncratic liver injury is unpredictable (10). In this case, infections such as hepatitis virus, cytomegalovirus, and rubella virus antibody were excluded, as were autoimmune diseases. According to the adverse reaction scoring principle of Naranjo's evaluation scale (11), the child's score information was 7 points, which determined the causality as probable.

Many studies have found that HLA genes are related to adverse reactions to many drugs (12). The protein encoded by the HLA gene is a cell surface receptor, which plays a key role in the immune system. Many studies have suggested that DILI may be related to HLA genotyping $(13,14)$. For example, HLA-B*5701 alleles are related to liver damage caused by flucloxacillin, and HLA-A*0201 and HLA-DQB1*0602 alleles are related to drug-induced liver damage caused by amoxicillin-clavulanate $(15,16)$. The gene HLA-A*02:06 is closely related to the adverse reactions caused by acetaminophen (17). However, further investigation is required to determine whether the allergic reaction and liver damage caused by ibuprofen is related to the HLA-B gene. This patient had obvious liver damage. After detecting this child's relevant HLA gene, the HLA-B ${ }^{\star} 5801$ test result was found to be positive. However, there is currently no evidence that there is a link between liver damage and SJS in children with this gene.

In summary, ibuprofen has a wide range of clinical applications, but it may cause serious adverse reactions among special populations. There are variations in reactions between different individuals. Especially in special populations with polymorphisms in the drug-gene, the probability of skin damage and liver damage after taking the 


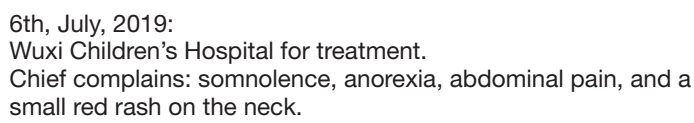

Wuxi Children's Hospital for treatment.

Chief complains: somnolence, anorexia, abdominal pain, and a small red rash on the neck.

8th, July, 2019:

Recurrent abdominal pain, rashes progressively worsening (involving the face, neck, and trunk) the skin tended to peel off

Treatment given: cefpiramide sodium, ornidazole, imipenem, cilastatin and gamma bulb.

$\checkmark$

11th, July, 2019:

Transferred to our hospital.

Chief complains: fever for 1 week, abdominal pain, rash, and abnormal liver function for 5 days

Diagnosis: liver insufficiency, Steven-Johnson syndrome, an intestinal infection.

Treatment given: methylprednisolone, loratadine, compound glycyrrhizin, reduced glutathione, adenosylmethionine succinate, ursodeoxycholic acid, Mupirocin ointment, gamma globulin, and albumin$$
\downarrow
$$

13th, July, 2019:

Liver function was aggravated by dynamic monitoring, the direct bilirubin was higher than before, and the yellowish skin was worse

Liver function: direct bilirubin, $169.69 \mu \mathrm{mol} / \mathrm{L}$; total bilirubin $187.07 \mu \mathrm{mol} / \mathrm{L}$; alanine aminotransferase, $283 \mathrm{U} / \mathrm{L}$; aspartate aminotransferase, $159 \mathrm{U} / \mathrm{L} ; \gamma$-glutamyltransferase, $437 \mathrm{U} / \mathrm{L}$; the total bile acid, $262 \mu \mathrm{mol} / \mathrm{L}$.

Treatment given: plasma exchange was given twice (July 13 to $14,1,400 \mathrm{~mL} /$ time)

31th, July, 2019:

Bilirubin and liver enzyme index decreased daily, exfoliative dermatitis gradually scabs healing

Drug-gene test:

Positive for HLA-B ${ }^{\star} 5801$

\section{3th, August, 2019:}

The patient was discharged after the condition gradually stabilized. No major compains on 15th and 30th day follow-up.

Examination findings: Direct bilirubin $13 \mu \mathrm{mol} / \mathrm{L}$, total bilirubin

$39.5 \mu \mathrm{mol} / \mathrm{L}$, alanine aminotransferase $159 \mathrm{U} / \mathrm{L}$, aspartate aminotransferase $56 \mathrm{U} / \mathrm{L}, \gamma$-glutamyltransferase $143 \mathrm{U} / \mathrm{L}$, total bile acid $63 \mu \mathrm{mol} / \mathrm{L}$

Figure 3 Progression of the case according to the time line. drug may be greatly increased. Clinical medical staff should strengthen their understanding of nonsteroidal drugs and monitor various biochemical indicators during medication time. Once abnormal damage occurs, it can be dealt with accordingly. At present, drug gene testing can be used as predictive biomarkers in clinical practice to avoid ADR. The high sensitivity (18) and specificity of some markers provide a credible basis for developing tests to identify individuals at risk of drug allergy. Drug-specific genetic screening tests can prevent the occurrence of such catastrophic diseases.

\section{Acknowledgments}

Funding: Shanghai Jiao Tong University Key Laboratory of Genetic Development and Mental and Neurological Diseases of Ministry of Education (No. 2020GDND01); Science Popularization Research Project of National Medical Economic Information Network of Chinese Pharmaceutical Association [No. CMEI2021KPYJ (JZYY) 00202]; Clinical Pharmacy Innovation Research Institute of Shanghai Jiao Tong University School of Medicine (No. CXYJY2019MS003).

\section{Footnote}

Reporting Checklist: The authors have completed the CARE reporting checklist. Available at http://dx.doi.org/10.21037/ tp-21-8

Peer Review File: Available at http://dx.doi.org/10.21037/tp21-8

Conflicts of Interest: All authors have completed the ICMJE uniform disclosure form (available at http://dx.doi. org/10.21037/tp-21-8). The authors have no conflicts of interest to declare.

Ethical Statement: The authors are accountable for all aspects of the work in ensuring that questions related to the accuracy or integrity of any part of the work are appropriately investigated and resolved. All procedures performed in studies involving human participants were in accordance with the ethical standards of the institutional and/or national research committee(s) and with the Helsinki 
Declaration (as revised in 2013). Written informed consent was obtained from the patient.

Open Access Statement: This is an Open Access article distributed in accordance with the Creative Commons Attribution-NonCommercial-NoDerivs 4.0 International License (CC BY-NC-ND 4.0), which permits the noncommercial replication and distribution of the article with the strict proviso that no changes or edits are made and the original work is properly cited (including links to both the formal publication through the relevant DOI and the license). See: https://creativecommons.org/licenses/by-nc-nd/4.0/.

\section{References}

1. Zoubek ME, Lucena MI, Andrade RJ, et al. Systematic review: ibuprofen-induced liver injury. Aliment Pharmacol Ther 2020;51:603-11.

2. Cac NN, Messingham MJ, Sniezek PJ, et al. StevensJohnson syndrome induced by doxycycline. Cutis 2007;79:119-22.

3. Harr T, French LE. Toxic epidermal necrolysis and Stevens-Johnson syndrome. Orphanet J Rare Dis 2010;5:39.

4. Caproni M, Torchia D, Schincaglia E, et al. Expression of cytokines and chemokine receptors in the cutaneous lesions of erythema multiforme and Stevens-Johnson syndrome/toxic epidermal necrolysis. Br J Dermatol 2006;155:722-8.

5. Yun J, Adam J, Yerly D, et al. Human leukocyte antigens (HLA) associated drug hypersensitivity: consequences of drug binding to HLA. Allergy 2012;67:1338-46.

6. Manson LEN, Swen JJ, Guchelaar HJ. Diagnostic Test Criteria for HLA Genotyping to Prevent Drug Hypersensitivity Reactions: A Systematic Review of Actionable HLA Recommendations in CPIC and DPWG Guidelines. Front Pharmacol 2020;11:567048.

7. Krebs K, Bovijn J, Zheng N, et al. Genome-wide Study Identifies Association between HLA-B*55:01 and Self-Reported Penicillin Allergy. Am J Hum Genet 2020;107:612-21.

8. Lee MH, Stocker SL, Anderson J, et al. Initiating

Cite this article as: Gui MZ, Ni M, Yin XD, Zhang T, Li ZL. Ibuprofen induced Stevens-Johnson syndrome and liver injury in children: a case report. Transl Pediatr 2021;10(6):1737-1742. doi: $10.21037 / \mathrm{tp}-21-8$ allopurinol therapy: do we need to know the patient's human leucocyte antigen status?. Intern Med J 2012;42:411-6.

9. Tassaneeyakul W, Jantararoungtong T, Chen P, et al. Strong association between HLA-B ${ }^{\star 5801}$ and allopurinolinduced Stevens-Johnson syndrome and toxic epidermal necrolysis in a Thai population. Pharmacogenet Genomics 2009;19:704-9.

10. European Association for the Study of the Liver. Electronic address: easloffice@easloffice.eu; Clinical Practice Guideline Panel: Chair:; Panel members; EASL Governing Board representative:. EASL Clinical Practice Guidelines: Drug-induced liver injury. J Hepatol 2019;70:1222-61.

11. Naranjo CA, Shear NH, Lanctôt KL. Advances in the diagnosis of adverse drug reactions. J Clin Pharmacol 1992;32:897-904.

12. Carr DF, Pirmohamed M. Biomarkers of adverse drug reactions. Exp Biol Med (Maywood) 2018;243:291-9.

13. Hussaini SH, Farrington EA. Idiosyncratic drug-induced liver injury: an update on the 2007 overview. Expert Opin Drug Saf 2014;13:67-81.

14. Grove JI, Aithal GP. Human leukocyte antigen genetic risk factors of drug-induced liver toxicology. Expert Opin Drug Metab Toxicol 2015;11:395-409.

15. Daly AK, Donaldson PT, Bhatnagar P, et al. HLA-B*5701 genotype is a major determinant of drug-induced liver injury due to flucloxacillin. Nat Genet 2009;41:816-9.

16. Lucena MI, Molokhia M, Shen Y, et al. Susceptibility to amoxicillin-clavulanate-induced liver injury is influenced by multiple HLA class I and II alleles. Gastroenterology 2011;141:338-47.

17. Ueta M, Nakamura R, Saito Y, et al. Association of HLA class I and II gene polymorphisms with acetaminophenrelated Stevens-Johnson syndrome with severe ocular complications in Japanese individuals. Hum Genome Var 2019;6:50.

18. Mallal S, Phillips E, Carosi G, et al. HLA-B*5701 screening for hypersensitivity to abacavir. N Engl J Med 2008;358:568-79.

(English Language Editors: J. Jones and J. Chapnick) 